\title{
On Rational Solutions of Yang-Baxter Equations. Maximal Orders in Loop Algebra
}

\author{
A. Stolin \\ Department of Mathematics, Stockholm University, Box 6701, S-11385 Stockholm, Sweden
}

Received February 6, 1991

\begin{abstract}
In 1982 Belavin and Drinfeld listed all elliptic and trigonometric solutions $X(u, v)$ of the classical Yang-Baxter equation (CYBE), where $X$ takes values in a simple complex Lie algebra $\mathfrak{g}$, and left the classification problem of the rational one open. In 1984 Drinfeld conjectured that if a rational solution is equivalent to a solution of the form $X(u, v)=C_{2} /(u-v)+r(u, v)$, where $C_{2}$ is the quadratic Casimir element and $r$ is a polynomial in $u, v$, then $\operatorname{deg}_{u} r=\operatorname{deg}_{v} r \leqq 1$. In another paper I proved this conjecture for $\mathfrak{g}=\mathfrak{s l}(n)$ and reduced the problem of listing "nontrivial" (i.e. nonequivalent to $C_{2} /(u-v)$ ) solutions of CYBE to classification of quasi-Frobenius subalgebras of $\mathfrak{g}$. They, in turn, are related with the so-called maximal orders in the loop algebra of $g$ corresponding to the vertices of the extended Dynkin diagram $D^{e}(\mathfrak{g})$. In this paper I give an algorithm which enables one to list all solutions and illustrate it with solutions corresponding to vertices of $D^{e}(\mathfrak{g})$ with coefficient 2 or 3 . In particular I will find all solutions for $\mathfrak{g}=\mathfrak{v}(5)$ and some solutions for $\mathfrak{g}=\mathfrak{o}(7), \mathfrak{v}(10), \mathfrak{o}(14)$ and $\mathfrak{g}_{2}$.
\end{abstract}

\section{Introduction}

This paper is a continuation of refs. [11-15]. I will recall, however, some of the notations and the main idea. In this paper I will explain how rational solutions of the classical Yang-Baxter equation (CYBE) for a simple complex Lie algebra $g$ correspond to the extended Dynkin diagram $D^{e}(\mathfrak{g})$. An announcement of the results of this paper had been delivered at the International Algebraic Conference in Novosibirsk, 1989 [13,14].

0.1. Formulation of the Problem. We will consider functions $X: \mathbb{C}^{2} \rightarrow \mathfrak{g} \otimes \mathfrak{g}$ such that

$$
\begin{gathered}
{\left[X^{12}\left(u_{1}, u_{2}\right), X^{13}\left(u_{1}, u_{3}\right)\right]+\left[X^{12}\left(u_{1}, u_{2}\right), X^{23}\left(u_{2}, u_{3}\right)\right]+\left[X^{13}\left(u_{1}, u_{3}\right), X^{23}\left(u_{2}, u_{3}\right)\right]=0,} \\
X^{12}(u, v)=-X^{21}(v, u), \quad(\mathrm{CYBE})
\end{gathered}
$$

and a solution will be called rational if it is of the form $X=C_{2} /(u-v)+r(u, v)$, where $r(u, v) \in \mathfrak{g}[u] \otimes \mathfrak{g}[v]$, cf. refs. $[2,3]$. 
Solutions $X_{1}$ and $X_{2}$ will be called gauge equivalent if there exists $\sigma(u) \in \operatorname{Aut} \mathrm{g}[u]$ such that $X_{1}=(\sigma \otimes \sigma) X_{2}$, where Aut $\mathrm{g}[u]$ is the group of automorphisms of $\mathrm{g}[u]$ considered as an algebra over $\mathbb{C}[u]$.

0.2. In [15] I proved the following two main theorems. First of all we need some notations. Denote by $\mathfrak{g}^{(1)}=\mathfrak{g} \otimes \mathbb{C}\left[u^{-1}, u\right]$ the loop algebra with the nondegenerate ad-invariant inner product $(x, y)=\operatorname{Res} \operatorname{tr}(\operatorname{ad} x$ ad $y)$.

Set $\mathbb{O}=\mathbb{C}\left[\left[u^{-1}\right]\right]$, the ring of formal power series in $u^{-1}, K=\mathbb{C}\left(\left(u^{-1}\right)\right)$, the field of quotients of $\boldsymbol{D}$.

Set $\mathfrak{g}(u)=\mathfrak{g} \otimes \mathbb{C}[u], \mathfrak{g}\left[\left[u^{-1}\right]\right]=\mathfrak{g} \otimes \mathbb{O}, \mathfrak{g}\left(\left(u^{-1}\right)\right)=\mathfrak{g} \otimes K$.

Theorem 1. There is a natural one-to-one correspondence between rational solutions of CYBE and subspaces $W \subset \mathfrak{g}\left(\left(u^{-1}\right)\right)$, such that

1) $W$ is a subalgebra in $\mathfrak{g}\left(\left(u^{-1}\right)\right)$ such that $W \supset u^{-N} \mathfrak{g}\left[\left[u^{-1}\right]\right]$;

2) $W \cap \mathfrak{g}[u]=\{0\}$ and $W \oplus \mathfrak{g}[u]=\mathfrak{g}\left(\left(u^{-1}\right)\right)$;

3) $W$ is Lagrangian with respect to the inner product in $\mathrm{g}\left(\left(u^{-1}\right)\right)$, i.e., $W^{\perp}=W$.

A $\mathbb{C}$-subalgebra $W \subset \mathfrak{g}\left(\left(u^{-1}\right)\right)$ such that $u^{K} \mathfrak{g}\left[\left[u^{-1}\right]\right] \supset W \supset u^{-N} \mathfrak{g}\left[\left[u^{-1}\right]\right]$ for some $K, N$ is called an order in $\mathfrak{g}\left(\left(u^{-1}\right)\right)$.

Theorem 2. Let $X_{1}$ and $X_{2}$ be rational solutions of CYBE. $W_{1}$ and $W_{2}$ the corresponding orders in $\mathrm{g}\left(\left(u^{-1}\right)\right)$. Then $X_{1}=(\sigma \otimes \sigma) X_{2} \leftrightarrow W_{1}=\sigma W_{2}$ for $\sigma(u) \in$ Aut $\mathfrak{g}[u]$.

0.3. Description of Orders in $\mathfrak{s l}(n)$.

Theorem. Any order in $\mathfrak{s l}(n ; K)$ is contained in $g^{-1} \mathfrak{s l}(n ; \mathbb{D}) g$ for some $g \in G L(n ; K)$.

Remark. Thus, any maximal order in $\mathfrak{s l}(n ; K)$ is an order of the form $g^{-1} \mathfrak{s l}(n ; \mathbb{D}) g$ for some $g \in G L(n ; K)$.

Sauvage Lemma [1]. The diagonal matrices $\operatorname{diag}\left(u^{m_{1}}, \ldots, u^{m_{n}}\right)$, where $m_{i} \in \mathbb{Z}$ for all $i, m_{1} \leqq \cdots \leqq m_{n}$ and $m_{i+1}-m_{i} \geqq-1$, represent all the double cosets $G L(u ; \mathbb{D}) \backslash$ $G L(n ; K) / G L(n ; \mathbb{C}[u])$.

Any order is contained in a maximal one. We need orders satisfying certain conditions. Taking Sauvage Lemma into account we reduce the problem of classification orders which correspond to rational solutions to the classification of maximal orders.

Clearly, $P G L(n, K)$ transitively acts on the set of maximal orders. It is easy to see that

$$
\operatorname{card}\{P G L(n, K) /(S L(n, K) / \text { center })\}=n .
$$

Indeed, $K^{*} /\left(K^{*}\right)^{n} \cong \mathbb{Z} / n \mathbb{Z}$. But $S L(n, K)$ does not transitively act on the set of maximal orders and there are $n$ representatives of this action. They are $d_{k}^{-1} \mathfrak{s l}(n, \mathbb{O}) d_{k}$, where $d_{k}=\operatorname{diag}(1, \ldots, 1, u, \ldots, u)$ with $k$-many 1 's.

The group $P G L(n, \mathbb{C}[u])=S L(n, \mathbb{C}[u]) /$ center acts on the set of maximal orders $\mathfrak{M} \subset \mathfrak{s l}(n, K)$ such that $\mathfrak{M}+\mathfrak{s l}(n, \mathbb{C}[u])=\mathfrak{s l}(n, K)$; there are $n$ orbits with the same representatives as above.

0.4. To an order contained in $d_{k}^{-1} \mathfrak{s l}(n, \mathbb{D}) d_{k}$ and satisfying conditions of Theorem 1 we assign a pair $(L, B)$, where $L+P_{k}=\mathfrak{s l}(n)$ for the parabolic subalgebra $P_{k}$ generated by all simple roots except the negative $k^{\text {th }}$ one and $B$ is a 2-cocycle on $L$ 
nondegenerate on $L \cap P_{k}$ (in other words, $L \cap P_{k}$ is a quasi-Frobenius subalgebra of $L$ ).

Making use of Elashvili's classification of Frobenius Lie algebras [5,6] and Spiz' classification of locally transitive irreducible linear Lie groups [10] I obtained (in principle) the classification of rational solutions of CYBE for $\mathfrak{s l}(n)$, see [15].

0.5 . Now, let us pass to an arbitrary finite-dimensional simple Lie algebra g. Let $G$ be the simply connected Lie group with Lie algebra g. Let $G(A)$ be the set of its $A$-points (the analog of $\mathfrak{s l}(n, A)$ ), $G_{\text {ad }}=G /$ center (in particular $G_{\text {ad }}(K)$ is the analog of $P G L(n, K))$. As is well-known ([17]), Aut $\mathrm{g}[u]=G_{\mathrm{ad}}(\mathbb{C}[u]) \cdot \Gamma$, where $\Gamma$ is a group of automorphisms of the Dynkin diagram $D(\mathrm{~g})$, and

$$
G_{\text {ad }}(\mathbb{C}[u])=G(\mathbb{C}[u]) / \text { center } G .
$$

\section{Maximal Orders in $\mathrm{g}\left(\left(u^{-1}\right)\right)$}

Let $\mathfrak{h}$ be a Cartan subalgebra in $\mathfrak{g}, R$ the set of roots of $\mathfrak{g}$. Put

$$
\mathfrak{h}_{\mathbb{R}}=\{h \in \mathfrak{h}: \alpha(h) \in \mathbb{R} \text { for any } \alpha \in R\} .
$$

Denote by $v: K \rightarrow \mathbb{Z}$ the valuation on $K$, i.e. the function such that

$$
v\left(\sum_{k \geqq N} a_{k} u^{-k}\right)=N \text {. }
$$

For $\alpha \in R$ and $h \in \mathfrak{h}_{\mathbb{R}}$ set

$$
\begin{aligned}
M_{\alpha}(h) & =\{f \in K: v(f) \geqq \alpha(h)\} ; \\
\mathbb{\Phi}_{h} & =\mathfrak{h}\left[\left[u^{-1}\right]\right] \oplus\left(\bigoplus_{\alpha \in R} M_{\alpha}(h) \mathfrak{g}_{\alpha}\right) .
\end{aligned}
$$

Clearly, $\mathbb{\Phi}_{h}$ is an order for any $h \in \mathfrak{h}_{\mathbb{R}}$ and since $M_{\alpha} M_{\beta} \subset M_{\alpha+\beta}$, the above representation of $\mathbb{O}_{h}$ is its $R$-grading.

Obviously,

$$
\mathbb{\Phi}_{h}+\mathfrak{g}[u]=\mathfrak{g}\left(\left(u^{-1}\right)\right) \Leftrightarrow X_{\alpha} u^{-\alpha(h)}+\mathbb{C}[u]=K \text { for any } \alpha \in R .
$$

Hence, $\alpha(h) \leqq 1$ but since $-\alpha \in R$, we have $|\alpha(h)| \leqq 1$ for any $\alpha \in R$.

The hyperplanes

$$
H_{\alpha, m}=\left\{h \in \mathfrak{h}_{\mathbb{R}}: \alpha(h)=m\right\}
$$

divide $\mathfrak{h}_{\mathbb{R}}$ into simplexes. Clearly,

$$
\mathbb{O}_{h}=\bigcap_{\substack{h_{i} \text { is a vertex of } \\ \text { the minimal symplex } \\ \text { containing } h}} \mathbb{\Phi}_{h_{i}} .
$$

1.1. Define the standard simplex setting

$$
\Delta_{s t}=\left\{h \in \mathfrak{h}_{\mathbb{R}}: \alpha(h) \geqq 0 \text { for all simple roots } \alpha \text { and } \alpha_{\max }(h) \leqq 1\right\} .
$$

Proposition. 1) $\mathbb{\Phi}_{h}$ is maximal $\Leftrightarrow h$ is a vertex of a simplex.

2) The vertices of $\Delta_{s t}$ correspond to maximal orders such that $\mathbb{\Phi}_{h}+\mathfrak{g}[u]=\mathfrak{g}\left(\left(u^{-1}\right)\right)$. 
The following Proposition 1.2 is borrowed from V. Drinfeld's correspondence with J.-P. Serre [8], cf. also [9].

1.2. Proposition. Any maximal order $\mathbb{D}$ such that $\mathbb{D}+\mathfrak{g}[u]=\mathfrak{g}\left(\left(u^{-1}\right)\right)$ is $G(\mathbb{C}[u])$ equivalent to a maximal order corresponding to a vertex of $\Delta_{s t}$.

More explicitly, the vertices of $\Delta_{s t}$ are: 0 and $h_{1}, \ldots, h_{r}$, where $r=r k g$. To every order $\mathbb{D}_{h}$ from Theorem 1 assign a vertex of the extended Dynkin diagram $D^{e}(\mathrm{~g})$ of $g$ according to the following rule:

$$
\begin{aligned}
& 0 \leftrightarrow \alpha_{\max } \\
& h_{i} \leftrightarrow \alpha_{i}, \quad \text { where } \alpha_{i}\left(h_{j}\right)=\delta_{i j} / k_{j} \text { and the } k_{j} \text { are to be } \\
& \text { found from the relation } \sum k_{j} \alpha_{j}=\alpha_{\max } .
\end{aligned}
$$

1.3. Proposition. Let $H \subset G$ be the Cartan subgroup, $H_{\mathrm{ad}}$ its image in $G_{\mathrm{ad}}, \alpha$ a vertex of $D(\mathfrak{g})$ such that $\sigma(\alpha)=\alpha_{\max }$ for some automorphism $\sigma \in \operatorname{Aut} D^{e}(\mathfrak{g})$. Then $\mathbb{\Phi}_{\alpha}=H_{\alpha}^{-1} \mathbb{D}_{0} H_{\alpha}$ for some $H_{\alpha} \in H_{\mathrm{ad}}(K)$.

Remarks. 1) If there exists an automorphism of the Dynkin diagram $D(\mathfrak{g})$ of $\mathfrak{g}$ sending $\alpha_{1}$ to $\alpha_{2}$, then the orders $\mathbb{D}_{\alpha_{1}}$ and $\boldsymbol{D}_{\alpha_{2}}$ are, clearly, gauge equivalent.

2) Let an order $W$ corresponding to a rational solution $C_{2} /(u-v)+r(u, v)$ be such that $W \subset \mathbb{D}_{0}$. Then $\operatorname{deg} r=0$.

Proposition 1.2 and Theorem 1 from Introduction enable one to prove Theorem 1.4 just as I have proved Drinfeld's conjecture for $\mathfrak{s l}(n)$ in [15].

1.4. Theorem. Drinfeld's conjecture is true for any $\mathfrak{g}$.

\section{Solutions Corresponding to Singular Vertices of $D^{e}(\mathbf{g})$}

A vertex of $D^{e}(\mathfrak{g})$ will be called singular if there exists an automorphism of $D^{e}(\mathfrak{g})$ sending the vertex to the vertex $\alpha_{\max }$ and regular otherwise.

Let $\alpha$ be a singular vertex of $D^{e}(\mathrm{~g}), P_{\alpha}$ the corresponding parabolic subalgebra of $\mathfrak{g}=\mathfrak{h} \oplus\left(\bigoplus_{\beta} \mathfrak{g}_{\beta}\right)$ :

$$
P_{\alpha}=\mathfrak{h} \oplus\left(\bigoplus_{\beta<0} \mathfrak{g}_{\beta}\right) \oplus\left(\bigoplus_{\substack{\gamma>0, \gamma \text { does not contain } \alpha \text { in the } \\ \text { decomposition w.r.t. simple roots }}} \mathfrak{g}_{\gamma}\right) .
$$

2.1. Theorem. The set of subalgebras $W \subset \mathbb{D}_{\alpha}$ satisfying conditions 1)-3) of Theorem 1 is in one-to-one correspondence with the set of pairs $(L, B)$ such that

1) $L \subset \mathfrak{g}$ is a Lie subalgebra and $L+P_{\alpha}=\mathfrak{g}$;

2) $B$ is a 2-cocycle on $L$ nondegenerate on $L \cap P_{\alpha}$.

2.2. Remarks. 1) Condition 1 of Theorem 2.1 is equivalent to the fact that $G(L)$ acts locally transitively on $G(\mathfrak{g}) / G\left(P_{\alpha}\right)$ and $1 \cdot G\left(P_{\alpha}\right)$ is a generic point of this action.

2) In order to list subalgebras $L$ in $\mathfrak{g}=\mathfrak{o}(n)$ satisfying condition 1) of Theorem 2.1 we can make use of the list of connected irreducible subgroups $G \subset S O(n ; \mathbb{C})$ locally transitive on the varieties $N_{k}$ of completely isotropic $k$-dimensional subspaces in $\mathbb{C}^{n}$, cf. [16] and Appendix 1. 
Proof of Theorem 2.1 is absolutely similar to that of Theorem 3.1 [15].

Many of the results of [15] apply to singular vertices of the extended Dynkin diagram of any simple $\mathfrak{g}$.

\section{Structure of Maximal Orders for Regular Vertices of the Extended Dynkin Diagram and the Corresponding Orders}

3.1. Let $\mathbb{\Phi}_{\alpha}$ be a maximal order where $\alpha$ is such that

let

$$
\alpha_{\max }=k \alpha+\sum_{\alpha_{i} \neq \alpha} k_{i} \alpha_{i}
$$

$$
L_{\alpha}=\mathfrak{h} \oplus\left(\underset{\beta}{\oplus} \mathfrak{g}_{\beta}\right), \quad \begin{aligned}
& \text { where in the expansion of } \beta \text { with respect to simple } \\
& \text { roots the coefficients of } \alpha \text { is either } 0 \text { or } \pm k
\end{aligned}
$$

(in other words, $L_{\alpha}$ is the semisimple Lie algebra whose Dynkin diagram is obtained from $D^{e}(\mathfrak{g})$ by discarding the vertex corresponding to $\alpha$ ). Let

$$
V_{\alpha, r}=\left\{\bigoplus_{\beta} E_{\beta}: \text { either } \beta=(k-r) \alpha+\sum_{\alpha \neq \alpha_{i}} k_{i} \alpha_{i}, 1 \leqq r<k \text { or } \beta=-r \alpha-\sum_{\alpha \neq \alpha_{i}} k_{i} \alpha_{i}\right\}
$$

let

$$
V_{\alpha}=\bigoplus_{r} V_{\alpha, r}
$$

Proposition. $\boldsymbol{\Phi}_{\alpha} / \boldsymbol{\Phi}_{\alpha}^{\perp} \cong\left(L_{\alpha}+\varepsilon^{k} L_{\alpha}\right) \oplus\left(\underset{r}{\oplus} \varepsilon^{r} V_{\alpha, r}\right)$, where $\varepsilon^{k+1}=0$.

3.1.1. Let

Set

$$
F_{i}(\varepsilon) \in\left(L_{\alpha}+\varepsilon^{k} L_{\alpha}\right) \oplus\left(\bigoplus_{r} \varepsilon^{r} V_{\alpha, r}\right) \quad \text { for } \quad i=1,2
$$

$$
\left(F_{1}, F_{2}\right)=\sum_{j+i=k}\left(A_{i}, A_{j}\right), \quad A_{i} \in \mathfrak{g} .
$$

Lemma. The inner product in $\mathfrak{g} \otimes K$ induces the inner product in $\mathbb{\Phi}_{\alpha} / \Phi_{\alpha}^{\perp}$ according to the formula (*).

3.2. Set

$$
\begin{aligned}
& P_{\alpha}^{ \pm}=\mathfrak{h} \oplus\left(\bigoplus_{\beta} \mathfrak{g}_{\beta}\right), \quad \begin{array}{l}
\text { where in the expansion of } \beta \text { with respect to simple } \\
\text { roots the coefficient of } \alpha \text { is either } 0 \text { or } \pm k,
\end{array} \\
& \text { respectively (in accordance with the superscript of } P_{\alpha}^{ \pm} \text {). }
\end{aligned}
$$

Set

and

$$
P_{\alpha, r}^{-}=\bigoplus_{\beta} \mathfrak{g}_{\beta}, \quad \text { where } \quad \beta=-r \alpha-\sum_{\alpha \neq \alpha_{i}} k_{i} \alpha_{i}
$$

$$
P_{\alpha, r}^{+}=\bigoplus_{\beta} \mathfrak{g}_{\beta}, \quad \text { where } \quad \beta=(k-r) \alpha+\sum_{\alpha_{i} \neq \alpha} k_{i} \alpha_{i}
$$


3.2.1. Proposition. There is a natural one-to-one correspondence between isotropic orders $W \subset \mathbb{D}_{\alpha}$ such that $W \oplus \mathfrak{g}[u]=\mathfrak{g} \otimes K$ and Lagrangian subalgebras

such that

$$
X_{W} \subset\left(L_{\alpha}+\varepsilon^{k} L_{\alpha}\right) \oplus\left(\bigoplus_{r} \varepsilon^{r} V_{\alpha, r}\right)
$$

$$
X_{W} \oplus\left(P_{\alpha}^{-}+\varepsilon^{k} P_{\alpha}^{-\perp}\right) \oplus\left(\bigoplus_{r} \varepsilon^{r} P_{\alpha, r}^{-}\right)=\left(L_{\alpha}+\varepsilon^{k} L_{\alpha}\right) \oplus\left(\bigoplus_{r} \varepsilon^{r} V_{\alpha, r}\right),
$$

where $P_{\alpha}^{-\perp}$ denotes the orthogonal complement of $P_{\alpha}^{-}$inside $L_{\alpha}$.

3.2.2. Proposition. Let $\mathfrak{g}$ be a simple Lie algebra, $\alpha$ a simple root entering with coefficient 2 in the decomposition of the maximal root. Let $X_{W}$ denote the same as in Proposition 3.2.1. Denote by $M$ the image of $X_{W}$ in $L_{\alpha}$ and define $V_{0} \subseteq V_{\alpha, 1}$ by $V_{0}=X_{W} \cap\left(\varepsilon V+\varepsilon^{2} L_{\alpha}\right) /\left(X_{W} \cap \varepsilon^{2} L_{\alpha}\right)$. Then

1) $M$ is a subalgebra of $L_{\alpha}$ such that $M+P_{\alpha}^{-}=L_{\alpha}$;

2) $V_{0}$ is a Lagrangian $M$-invariant subspace in $V_{\alpha, 1}$.

Remark. If $V_{0}=P_{\alpha, 1}^{+}$then the corresponding solution, if any, is a constant one.

\section{Calculations}

The following two Lemmas accumulate certain well-known facts (deducible for instance from [7]) in a form needed in the sequel.

4.1. Lemma. $L_{\alpha}$ is a semisimple Lie algebra whose Dynkin diagram is obtained from $D^{e}(\mathrm{~g})$ by a deleting the vertex corresponding to $\alpha$ (and the segments connected with it); $P_{\alpha}^{-}$is a parabolic subalgebra of $L_{\alpha}$.

4.2. Lemma. For the series $\mathrm{B}, \mathrm{C}, \mathrm{D}$ we have:

B) $L_{\alpha}=\mathfrak{o}(2 n+1) \oplus \mathfrak{o}(2 m), \quad V_{\alpha}=\mathbb{C}^{2 n+1} \otimes \mathbb{C}^{2 m} ; \quad m \geqq 2 ;$

C) $L_{\alpha}=\mathfrak{s p}(2 n) \oplus \mathfrak{s p}(2 m), \quad V_{\alpha}=\mathbb{C}^{2 n} \otimes \mathbb{C}^{2 m} ; \quad n \geqq 2 ; \quad n \geqq 2 ;$

D) $L_{\alpha}=\mathfrak{o}(2 n) \oplus \mathfrak{o}(2 m), \quad V_{\alpha}=\mathbb{C}^{2 n} \otimes \mathbb{C}^{2 m} ; \quad m \geqq 2 ; \quad n \geqq 2$;

and in all the cases $V_{\alpha}$ is an irreducible $L_{\alpha}$-module with an invariant symmetric inner product induced by the Killing form.

4.3. Proposition. Let $\mathrm{g}$ be a simple Lie algebra of series $\mathrm{B}-\mathrm{D}, \alpha \in D(\mathrm{~g})$ a regular vertex, $M \subset L_{\alpha}$ a subalgebra satisfying conditions of Proposition 3.2.2. Then $M$ is contained in a parabolic subalgebra of the semisimple Lie algebra $L_{\alpha}$.

4.4.1. Proposition. Let $\mathfrak{g}=\mathfrak{o}(2 n+1)$. If $W \subset \mathbb{O}_{\alpha_{n}}$ then there exists a gauge transformation $\sigma(u) \in$ Aut $\mathrm{g}[u]$ such that either $\sigma(u) W \subset \mathbb{D}_{0}$ or $\sigma(u) W \subset \mathbb{O}_{\alpha_{1}}$.

Recall that the Dynkin diagram of $\mathfrak{o}(2 n+1)$ is of the form

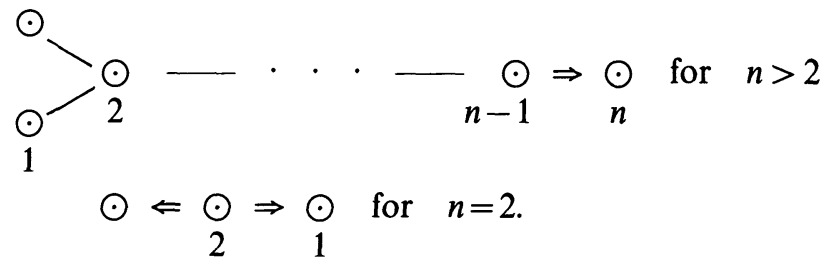


4.5. Proposition. Let $\alpha_{1}$ be the singular vertex of $D(\mathfrak{o}(2 n+1))$. Let $(L, B)$ determine a solution in $\mathbb{D}_{\alpha_{1}}$ and there exists an L-invariant $r$-dimensional isotropic subspace in $\mathbb{C}^{2 n+1}$. Then

1) if $r=1$ this solution is gauge equivalent to a constant one;

2) if $r>1$ this solution is gauge equivalent to a solution from $\boldsymbol{\Phi}_{\alpha_{r}}$.

4.6. Corollary. 1) There is a unique nonconstant solution corresponding to the singular vertex $\alpha_{1} \in D(\mathfrak{o}(5))$ and an irreducible $L \subset \mathfrak{o}(5)$. In this case $L=\mathfrak{o}(3), B=0$ and $\mathfrak{D}(3)$ is realized as a subalgebra of $\mathrm{o}(5)$ according to the embedding $R\left(4 \Lambda_{1}\right): \mathfrak{o}(3) \rightarrow \mathfrak{o}(5)$. (We use notations from [16].)

2) Besides this there are 3 nonconstant solutions. They correspond to $\alpha_{1} \in D(\mathfrak{o}(5))$ and to the following subalgebras $L \subset \mathfrak{o}(5)$ :

a)

b)

$$
L=\left(\begin{array}{lllll}
* & * & * & * & 0 \\
* & * & * & 0 & * \\
0 & 0 & 0 & * & * \\
0 & 0 & 0 & * & * \\
0 & 0 & 0 & * & *
\end{array}\right)
$$

c)

$$
L=g^{-1}\left(\begin{array}{lllll}
* & * & 0 & * & 0 \\
* & * & 0 & 0 & * \\
0 & 0 & 0 & 0 & 0 \\
0 & 0 & 0 & * & * \\
0 & 0 & 0 & * & *
\end{array}\right) g
$$

where $g \in S O(5)$ is such that $g\left(\begin{array}{l}0 \\ 0 \\ 0 \\ 0 \\ 1\end{array}\right)=\left(\begin{array}{c}1 \\ 0 \\ \sqrt{2} \\ 1 \\ -1\end{array}\right)$

Remark. Here $\mathfrak{o}(n)$ preserves the following symmetric form on $\mathbb{C}^{n}: B=x_{1} y_{n}+$ $x_{2} y_{n-1}+\cdots+x_{n} y_{1}$.

\section{Solutions of CYBE Corresponding to a Simple Root of $D^{e}(\mathbf{g})$ with Coefficient 3}

5.1. Lemma. Let $\alpha$ be a simple root whose coefficient in the decomposition of the maximal root with respect to simple ones is 3 . In notations of Sect. 3 the $L_{\alpha}$-module $V_{\alpha}$ is of the form $V_{\alpha, 1} \oplus V_{\alpha, 2}$, where $V_{\alpha, 1}$ and $V_{\alpha, 2}$ are irreducible $L_{\alpha}$-modules and $\left[V_{\alpha, 1}, V_{\alpha, 1}\right] \subset V_{\alpha, 2}$. 
5.2. Let $L$ be an arbitrary Lie algebra; $V$ a vector space and $f: L \rightarrow \mathfrak{g l}(V)-$ a representation. Denote by $(L, V)$ the Lie algebra whose space is $L \oplus V$ and bracket is given by the formula:

$$
\left[\left(l_{1}, v_{1}\right),\left(l_{2}, v_{2}\right)\right]_{\mathrm{af}}=\left(\left[l_{1}, l_{2}\right], f\left(l_{1}\right) v_{2}-f\left(l_{2}\right) v_{1}\right) .
$$

We will call $(L, V)$ an affine Lie algebra.

5.2.1. Proposition. Let $\alpha$ be a simple root from Lemma 5.1. Let $X_{W}$ be a Lagrangian subalgebra satisfying conditions of Proposition 3.2.1. Then there is a natural 1-1 correspondence between the set of such subalgebras and the set of pairs $(S, B)$, where:

1) $S$ is a subalgebra of $\left(L_{\alpha}, V_{\alpha, 1}\right)$ such that

$$
S+\left(P_{\alpha}^{-}, P_{\alpha, 1}^{-}\right)=\left(L_{\alpha}, V_{\alpha, 1}\right)
$$

2) B is a skewsymmetric 2-form on $S$, nondegenerate on $S \cap\left(P_{\alpha}^{-}, P_{\alpha, 1}^{-}\right)$and such that

$$
B\left([x, y]_{\mathrm{af}}, z\right)+B\left([z, x]_{\mathrm{af}}, y\right)+B\left([y, z]_{\mathrm{af}}, x\right)=([x, y], z)
$$

for any $x, y, z \in S$.

Here in the expression $([x, y], z)$ we consider $x, y, z$ as elements of $L_{\alpha}+\varepsilon V_{\alpha, 1}+$ $\varepsilon^{2} V_{\alpha, 2}+\varepsilon^{3} L_{\alpha}$.

5.2.2. Lemma. There is a 1-1 correspondence between the set of subalgebras of the affine Lie algebra $(L, V)$ and the set of triples $\left(L_{1}, V_{1}, r\right)$ where:

1) $L_{1}$ is a subalgebra in $L$;

2) $V_{1}$ is an $L_{1}$-submodule in $V$;

3) $r$ is a 1-cocycle on $L_{1}$ with values in $V / V_{1}$.

Since there are finitely many roots corresponding to vertices of $D^{e}(\mathfrak{g})$ satisfying Lemma 5.1, it is possible to investigate all such cases.

5.3. Corollary. Let $\mathfrak{g}=\mathrm{g}_{2}$; there is only one $\alpha$ as in Lemma 5.1 and for it we have $L_{\alpha} \cong \mathfrak{s l}(3), V_{\alpha, 1} \cong \mathbb{C}^{3}, P_{\alpha}^{-}=P_{2}$ (in notations of $\left.[15]\right), P_{\alpha, 1}^{-}=\mathbb{C}^{2}$. There exists $a$ solution in this case with $L=\mathfrak{D}(3) \subset\left(\mathfrak{s l}(3), \mathbb{C}^{3}\right)$ given by the formula:

$$
L=\left\{(T)\left(\begin{array}{ccc}
a & b & 0 \\
c & 0 & -b \\
0 & -c & -a
\end{array}\right)(T)^{-1},\left(\begin{array}{c}
0 \\
-b \\
-a
\end{array}\right)\right\}
$$

where

$$
(T)=\left(\begin{array}{lll}
1 & 0 & 0 \\
0 & 1 & 0 \\
1 & 1 & 1
\end{array}\right)
$$

In the following cases 5.4-5.7 I have only calculated all the data but not the solutions themselves, if any.

5.4. Corollary. $\mathfrak{g}=\mathfrak{f}_{4}$; there is only one $\alpha$ as in Lemma 5.1 and for it we have

$$
\begin{aligned}
L_{\alpha} & =\mathfrak{s l}(3) \oplus \mathfrak{s l}(3) ; \quad V_{\alpha}=\mathbb{C}^{3} \otimes \mathbb{C}^{6} \quad\left(=R\left(\Lambda_{1}\right) \otimes R\left(2 \Lambda_{1}\right)\right), \\
P_{\alpha}^{-} & =P_{2} \oplus \mathfrak{s l}(3) ; \quad P_{\alpha, 1}^{-}=\mathbb{C}^{12} .
\end{aligned}
$$


5.5. Corollary. $\mathfrak{g}=\mathfrak{e}_{8}$. There are two roots satisfying Lemma 5.1, let us denote them by $\alpha_{1}$ and $\alpha_{2}$ :

1) $\alpha=\alpha_{1}$. Then



2) $\alpha=\alpha_{2}$. Then

$$
L_{\alpha}=\mathfrak{s l}(9) ; \quad V_{\alpha}=\mathbb{C}^{84}\left(=R\left(\Lambda_{3}\right)\right) ; \quad P_{\alpha}^{-1}=P_{8} ; \quad P_{\alpha, 1}^{-}=\mathbb{C}^{56}
$$

$L_{\alpha}=\mathrm{e}_{6} \oplus \mathfrak{s l}(3) ; \quad V_{\alpha}=\mathbb{C}^{27} \otimes \mathbb{C}^{3}\left(=R\left(\Lambda_{1}\right) \otimes R\left(\Lambda_{1}\right)\right) ; \quad P_{\alpha}^{-}=\mathrm{e}_{6} \oplus P_{2} ; \quad P_{\alpha, 1}^{-}=\mathbb{C}^{54}$.

5.6. Corollary. $\mathfrak{g}=\mathfrak{e}_{6}$. There is one root satisfying Lemma 5.1, let us denote it by $\alpha_{1}$

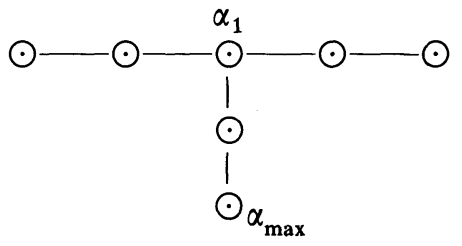

Then $L_{\alpha}=\mathfrak{s l}(3) \oplus \mathfrak{s l}(3) \oplus \mathfrak{s l}(3), V_{\alpha}=\mathbb{C}^{27}\left(=R\left(\Lambda_{1}\right) \times R\left(\Lambda_{1}\right) \times R\left(\Lambda_{1}\right)\right)$,

$$
P_{\alpha}^{-}=P_{2} \oplus \mathfrak{s l}(3) \oplus \mathfrak{s l}(3) . \quad P_{\alpha, 1}^{-}=\mathbb{C}^{18} .
$$

5.7. Corollary. $\mathfrak{g}=\mathrm{e}_{7}$. There are two roots satisfying Lemma 5.1. Let us denote them by $\alpha_{1}$ and $\alpha_{2}$,

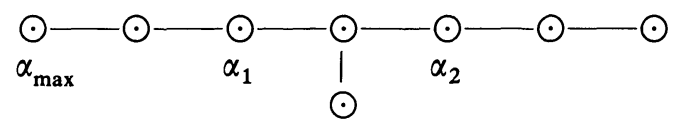

Then in both cases $L_{\alpha}=\mathfrak{s l}(3) \oplus \mathfrak{s l}(6), V_{\alpha}=\mathbb{C}^{45}\left(R\left(\Lambda_{1}\right) \times R\left(\Lambda_{2}\right)\right)$.

\section{Appendix 1}

List of Connected Subgroups $G \subset S O(n)$ Locally Transitive on the Grassman Manifold $G_{k}^{n}$ of Isotropic $k$-Dimensional Subspaces

Table 1

\begin{tabular}{lll}
\hline$G$ & Embedding & $k$ \\
\hline$S O(n), n \geqq 3$ & id & $1,2, \ldots,[n / 2]^{(2)}$ \\
$S p(2 s) \times S L(2), s \geqq 4$ & $\Lambda_{1} \otimes \Lambda_{1}$ & $1,2,3,2 s^{(1)}$ \\
$S p(6) \times S L(2)$ & $\Lambda_{1} \otimes \Lambda_{1}$ & $1,2,3,5,6^{(2)}$ \\
$S p(4) \times S L(2)$ & $\Lambda_{1} \otimes \Lambda_{1}$ & $1,2,3,4^{(2)}$ \\
$G_{2}$ & $\Lambda_{1}$ & $1,2,3$ \\
$\operatorname{Spin}(7)$ & $\Lambda_{3}$ & $1,2,3,4^{(2)}$ \\
$\operatorname{Spin}(9)$ & $\Lambda_{4}$ & $1,2,3,8^{(1)}$ \\
$S L(3)$ & $\mathrm{Ad}$ & $1,4^{(2)}$ \\
$S p(4)$ & $2 \Lambda_{1}$ & $1,5^{(2)}$ \\
$G_{2}$ & $\Lambda_{2}$ & 1 \\
$S O(3)$ & $4 \Lambda_{1}$ & 1,2 \\
$S p(6)$ & $\Lambda_{2}$ & $1,7^{(2)}$ \\
$F_{4}$ & $\Lambda_{1}$ & 1 \\
$S p(2 s) \times S p(4), s \geqq 2$ & $\Lambda_{1} \otimes \Lambda_{1}$ & 1 \\
\hline
\end{tabular}


$\mathrm{Sign}^{(2)}$ means that $G$ acts locally transitively on the both connected components of $G_{n}^{2 n}$.

$\mathrm{Sign}^{(1)}$ means that $G$ acts locally transitively only on one connected component of $G_{n}^{2 n}$.

The list given by Table 1 is borrowed from [16].

\section{A.1.1. Lemma}

1) Let $\odot \alpha_{\max }$<smiles></smiles>

be the extended Dynkin diagram of $\mathrm{o}(2 n+1)$. To find solutions corresponding to $\boldsymbol{\Phi}_{\alpha_{1}}$ we have to take $k=1$ in Table 1.

2)

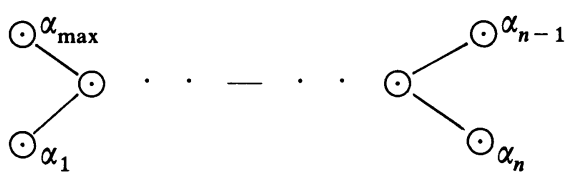

be the extended Dynkin diagram of $\mathrm{o}(2 n)$. To find solutions corresponding to $\mathbb{\Phi}_{\alpha_{1}}$ we have to take $k=1$ in Table 1 .

Since the orders $\mathbb{\Phi}_{\alpha_{n-1}}$ and $\mathbb{\Phi}_{\alpha_{n}}$ are gauge equivalent, we have to take $k=n$ in order to find the corresponding solutions.

A.1.2. Examples of Solutions. The following 3 cases from Table 1 are the only ones with the trivial stationary subgroup of a generic point and to each of them there corresponds exactly one solution.

\begin{tabular}{llll}
\hline $\mathfrak{g}$ & $L$ & $k$ & Embedding \\
\hline $\mathfrak{o}(5)$ & $\mathfrak{s l}(2)$ & 1 & $4 \Lambda_{1}$ \\
$\mathfrak{o}(10)$ & $\mathfrak{s p}(4)$ & 5 & $2 \Lambda_{1}$ \\
$\mathfrak{o}(14)$ & $\mathfrak{s p}(5)$ & 7 & $\Lambda_{2}$ \\
\hline
\end{tabular}

A.2. Example $\mathfrak{g}=\mathfrak{o}(7)$.

Recall that $D^{e}(\mathrm{~g})$ is

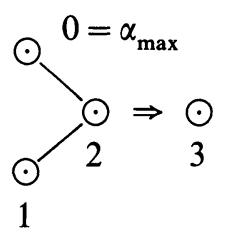

Proposition. 1) There are no solutions corresponding to $\mathbb{\Phi}_{1}$ such that the corresponding subalgebra $L \subset \mathfrak{o}(7)$ is irreducible.

2) If a solution corresponds to $\mathbb{\Phi}_{1}$ and a subalgebra $L \subset \mathfrak{o}(7)$ is such that there 
exists an L-invariant 1-dimensional isotropic subspace, then the solution is gauge equivalent to a constant one.

3) There exists a solution corresponding to $\boldsymbol{\Phi}_{1}$ given by the following subalgebra preserving a 2-dimensional isotropic subspace:

$$
L=\left\{\left(\begin{array}{ccccccc}
a+t & b & * & * & * & * & 0 \\
c & -a+t & * & * & * & 0 & * \\
0 & 0 & a & b & 0 & * & * \\
0 & 0 & c & 0 & -b & * & * \\
0 & 0 & 0 & -c & -a & * & * \\
0 & 0 & 0 & 0 & 0 & a-t & -b \\
0 & 0 & 0 & 0 & 0 & -c & -a-t
\end{array}\right)\right\}
$$

Remark. Here $\mathfrak{o}(n)$ preserves the following symmetric form on $\mathbb{C}^{n}$ :

$$
B=x_{1} y_{n}+x_{2} y_{n-1}+\cdots+x_{n} y_{1} \text {. }
$$

\section{Proofs}

6.0. Proof of Proposition 1.3. Consider the automorphism $\varphi$ of $\mathfrak{g} \otimes K=\mathfrak{g}\left(\left(u^{-1}\right)\right)$ such that $\varphi=i d$ on $\mathfrak{h}$ and for every $\operatorname{root} \beta$ the restriction of $\varphi$ to $\mathfrak{g}_{\beta}$ equals $u^{k(\beta)}$, where $k(\beta)$ is the coefficient of $\alpha$ in the decomposition of $\beta$ with respect to simple roots. Then $\mathbb{D}_{\alpha}=\varphi^{-1}\left(\mathbb{D}_{0}\right)$ (to prove this notice that according to [4] the coefficient of $\alpha$ in the decomposition of $\alpha_{\max }$ equals 1). Clearly, $\varphi$ corresponds to some $H_{\alpha} \in H_{\text {ad }}(K)$. Then $\mathbb{D}_{\alpha}=H_{\alpha}^{-1} \mathbb{D}_{0} H_{\alpha}$.

6.1. Proof of Theorem 2.1. By Proposition 1.3 there exists $H_{\alpha} \in H_{\mathrm{ad}}(K)$ such that $W \subset H_{\alpha}^{-1} \mathbb{D}_{0} H_{\alpha}=\mathbb{D}_{\alpha}$. Therefore $W^{\perp}=W \supset H_{\alpha}^{-1}\left(u^{-2} \mathbb{D}_{0}\right) H_{\alpha}=u^{-2} \mathbb{D}_{\alpha}$.

Similarly to [15] we can prove the following lemma and the Proposition 6.1.2 it implies.

\subsubsection{Lemma}

$$
\mathbb{D}_{\alpha} / u^{-2} \boldsymbol{D}_{\alpha} \cong \mathfrak{g} \otimes\left(\mathbb{C}[\varepsilon] /\left(\varepsilon^{2}\right)\right) .
$$

6.1.2. Proposition. There is a natural one-to-one correspondence between orders $W \subset \mathbb{O}_{\alpha}$ satisfying the conditions of Theorem 2.1 and Lagrangian subalgebras $X_{W} \subset \mathfrak{g}(\mathbb{C}[\varepsilon])$ such that $X_{W} \oplus\left(P_{\alpha}+\varepsilon P_{\alpha}^{\perp}\right)=\mathfrak{g}(\mathbb{C}[\varepsilon])$.

Now it is absolutely evident that the proof of Theorem 2.1 is absolutely similar to that of Theorem 3.1 from [15].

6.2. For maximal orders corresponding to singular vertices a lot of results from [15] are generalized practically literally.

6.2.1. Lemma. Let $\left(L_{1}, B_{1}\right)$ and $\left(L_{2}, B_{2}\right)$ determine solutions from $\boldsymbol{D}_{\alpha}$. Let $(\operatorname{Ad} X)\left(L_{1}\right)=L_{2}$ and $B_{2}\left(\operatorname{Ad} X\left(a_{1}\right), \operatorname{Ad} X\left(a_{2}\right)\right)=B_{1}\left(a_{1}, a_{2}\right)$ for any $a_{1}, a_{2} \in L$ and some $X \in \operatorname{Ad} G\left(P_{\alpha}\right)$. Then the corresponding solutions are gauge equivalent.

6.2.2. Proposition. Let $\left(L, B_{1}\right)$ and $\left(L, B_{2}\right)$ determine solutions corresponding to $\boldsymbol{D}_{\alpha}$. Let $B_{1}$ be cohomologic to $B_{2}$. Then these solutions are gauge equivalent. 
6.2.3. Lemma. Let $L \cap P_{\alpha}$ be a Frobenius Lie algebra. Then every class of $H^{2}(L)$ contains a representative nondegenerate on $L \cap P_{\alpha}$.

6.2.4. Proposition. Let $L$ satisfy the conditions of Lemma 6.2.3 and $H^{2}(L)=0$. Then there is only one solution corresponding to $\mathbb{\Phi}_{\alpha}$ with given $L$.

Thanks to Lemma 6.1.1 and Proposition 6.1.2 all proofs are identical to the corresponding ones for $\mathfrak{g}=\mathfrak{s l}(n)$.

\section{Proof of Statements from Section 3}

7.1. Proof of Proposition 3.1. Let $R$ be the set of all roots. Set

$$
R_{r}=\left\{\beta \in R: \beta=r \alpha+\sum_{\alpha_{i} \neq \alpha} k_{i} \alpha_{i}\right\} ; \quad g_{0}=\mathfrak{h} \otimes \sum_{\alpha \in R_{0}} \mathfrak{g}_{\alpha} ; \quad \mathfrak{g}_{r}=\sum_{\alpha \in R_{r}} \mathfrak{g}_{\alpha} .
$$

The statement of the proposition follows from equalities:

$$
\begin{aligned}
& \Phi_{\alpha}=\sum_{r=1}^{k} u^{-1} \Phi g_{r}+\sum_{r=1-k}^{0} \Phi g_{r}+u \Phi g_{-k} \\
& \Phi_{\alpha}^{\perp}=\sum_{r=-k}^{1} u^{-1} \Phi g_{r}+\sum_{r=0}^{k-1} u^{-2} \Phi g_{r}+u^{-3} \Phi g_{k}
\end{aligned}
$$

7.1.1. Proof of Lemma 3.1.1. Consider the form $(a, b)=\operatorname{Tr} a b$ on $\mathfrak{g} \subset \mathfrak{s l}(k)$.

Clearly, $V_{\alpha, r_{1}}$ is orthogonal to $V_{\alpha, r_{2}}$ if $r_{1}+r_{2} \neq k$ and the pairing is nondegenerate if $r_{1}+r_{2}=k$. This implies the statement of the lemma.

7.2.1. Proof of Proposition 3.2.1. The statement follows from the fact that the projection of $\boldsymbol{\Phi}_{\alpha}$ to $\left(L_{\alpha}+\varepsilon^{k} L_{\alpha}\right) \oplus \sum_{r} \varepsilon^{r} V_{\alpha, r}$ maps $\mathfrak{g}[u] \cap \boldsymbol{\Phi}_{\alpha}$ precisely onto $\left(P_{\alpha}+\varepsilon^{k} P_{\alpha}^{\perp}\right) \oplus \sum_{r} \varepsilon^{r} P_{\alpha, r}^{-}$.

\subsubsection{Proof of Proposition 3.2.2.}

1) Let $X_{W}$ satisfy the conditions of the proposition. Let $M$ be the image of $X_{W}$ under the projection

$$
L_{\alpha}+\varepsilon V_{\alpha, 1}+\varepsilon^{2} L_{\alpha} \rightarrow L_{\alpha}
$$

From Proposition 3.2.1, it is clear that $M+P_{\alpha}^{-}=L_{\alpha}$.

2) Let $V$ be a linear vector space over $\mathbb{C}$ with a nondegenerate inner product. Let $S$ be an isotropic subspace. Thus, $S^{\perp} \supset S$ and $B$ determines a nondegenerate inner product on $S^{\perp} / S$.

Lemma 1. Let $V$ and $S$ be as above and $X-a$ Lagrangian subspace in $V$. Then $\left(X \cap S^{\perp}\right) /(X \cap S)$ is a Lagrangian subspace in $S^{\perp} / S$.

Proof. Clearly, $\left(X \cap S^{\perp}\right) /(X \cap S)$ is an isotropic subspace in $S^{\perp} / S$. Then dimensional considerations show that $\left(X \cap S^{\perp}\right) /(X \cap S)$ is a Lagrangian subspace. The lemma is proved.

The following lemma highlights the structure of Lagrangian subspaces in a direct sum of modules. 
Lemma 2. Let $V=V_{1} \oplus V_{2}$ be a linear vector space with a nondegenerate inner product and $V_{1}^{\perp}=V_{2}$. Then there exists a one-to-one correspondence Lagrangian subspace in $V\} \leftrightarrow\left\{\left(S_{1}, S_{2}, \Phi\right): S_{i}\right.$ is an isotropic subspace in $V_{i} ; \Phi: S_{1}^{\perp} / S_{1} \rightarrow S_{2}^{\perp} / S_{2}$ is an isomorphism such that $(x, y)=-(\Phi(x), \Phi(y))\}$.

Remark. We denoted by $S_{i}^{\perp} \subset V_{i}$ the set $\left\{x \in V_{i}:\left(x, S_{i}\right)=0\right\}$.

Proof. Given a triple $\left\{S_{1}, S_{2}, \Phi\right\}$. We see that $X=\left\{s_{1}^{\perp}+s_{1}+\Phi\left(s_{1}\right)+S_{2}^{\perp}: s_{i}^{\perp} \in S_{i}^{\perp}\right.$; $\left.s_{1} \in S_{1}\right\}$ is a Lagrangian subspace.

Conversely, given $X$. We see that $S_{i}=X \cap V_{i}$ is an isotropic subspace in $V_{i}$. Then $S_{i}^{\perp} \subset V_{i}$ is the image of $X$ in $V_{i}$ under the projection $V \rightarrow V_{i}$ and one has two exact sequences $0 \rightarrow S_{2} \rightarrow X \rightarrow S_{1}^{\perp} \rightarrow 0$ and $0 \rightarrow S_{1} \rightarrow X \rightarrow S_{2}^{\perp} \rightarrow 0$. Thus, there exists an isomorphism $\Phi: S_{1}^{\perp} / S_{1} \rightarrow S_{2}^{\perp} / S_{2}$. Obviously, $(\Phi(x), \Phi(y))=-(x, y)$. The lemma is proved.

Let $X_{W}$ be a Lagrangian subalgebra in $L_{\alpha}+\varepsilon^{2} L_{\alpha}+\varepsilon V . X_{W} \cap\left(\varepsilon V+\varepsilon^{2} L_{\alpha}\right) /$ $\left(X_{W} \cap \varepsilon^{2} L_{\alpha}\right)$ is a Lagrangian subspace in $\left(\varepsilon V+\varepsilon^{2} L_{\alpha}\right) / \varepsilon^{2} L_{\alpha}=\varepsilon V$ by Lemma 1 .

We have recovered from $X_{W}$ a subalgebra in $L_{\alpha}+\varepsilon^{2} L_{\alpha}$ and a Lagrangian submodule $V_{0}$ in $\varepsilon V$. Since $X_{W} \cap \varepsilon^{2} L_{\alpha}$ and $X_{W} \cap\left(\varepsilon V+\varepsilon^{2} L_{\alpha}\right)$ are ideals in $X$, then, clearly, $V_{0}$ is an $M$-module.

The proposition is proved.

8.1. Proof of Proposition 4.3. For definiteness sake consider the case $\mathfrak{o}(k) \oplus \mathfrak{o}(2 n)$. Suppose $M \subset L_{\alpha}$ cannot be embedded into any parabolic subalgebra of $L_{\alpha}$. Let us prove that $M$ is semisimple. If $M$ acts irreducibly on $\mathbb{C}^{k} \otimes \mathbb{C}^{2 n}$, then $M$ is semisimple (it cannot be reductive since the scalar matrices do not preserve the inner product).

Suppose that $V$ is an $M$-invariant subspace in $\mathbb{C}^{k} \otimes \mathbb{C}^{2 n}$. Clearly, the inner product on $V$ must be nondegenerate (if there exists $V_{0} \subset V$ such that $\left(V_{0}, V\right)=0$, then choosing a maximal $V_{0}$ we get $\left(M V_{0}, V\right)+\left(V_{0}, M V\right)=0$, hence $M V_{0} \subset V_{0}$ and $V_{0}$ is isotropic, i.e. $M$ is embedded into a parabolic subalgebra).

Then $V^{\perp}$ is $M$-invariant and $V \oplus V^{\perp}=\mathbb{C}^{k} \otimes \mathbb{C}^{2 n}$. This implies that $M$ is reductive. Having considered irreducible components on which the inner product is nondegenerate we see that $M$ is centerless, since the matrices acting by multiplication by a scalar do not preserve the inner product.

Thus, $M$ is semisimple. Let us consider the projection of $M$ into $\mathfrak{o}(k)$. The representation of $M$ in $\mathbb{C}^{k}$ can be decomposed into the direct sum of irreducible components, the latter being distinct, otherwise $M$ can be embedded in a parabolic subalgebra.

First, let us consider a particular case when the representations of $M$ in $\mathbb{C}^{k}$ and in $\mathbb{C}^{2 n}$ are irreducible.

Let $\lambda$ and $\mu$ respectively be their highest weights. Since in $\mathbb{C}^{k}$ there exists a nondegenerate invariant inner product, then $\omega_{0}(\lambda)=-\lambda$, cf. ref. [4] (8.7.5.).

Analogously, $\omega_{0}(\lambda)=-\lambda$.

There exists an $M$-submodule $X$ in $\mathbb{C}^{k} \otimes \mathbb{C}^{2 n}$ having $\lambda+\mu$ as its highest weight and irreducible.

Since $\omega_{0}(\lambda+\mu)=-\lambda-\mu$, then the inner product in $X$ is nondegenerate.

In this case the decomposition of $\mathbb{C}^{k} \otimes \mathbb{C}^{2 n}$ in irreducible components cannot contain more than one component isomorphic to $X$, because $\lambda$ and $\mu$ are unique. Thus, $\mathbb{C}^{k} \otimes \mathbb{C}^{2 n}$ cannot contain a Lagrangian $M$-module by Lemma 2 (cf. item 7.2.1).

- The case of $M$ reducibly acting in $\mathbb{C}^{k}$ and $\mathbb{C}^{2 n}$ is immediately reduced to an 
irreducible case since we can choose the greatest of the highest weights in $\mathbb{C}^{k}$ and $\mathbb{C}^{2 n}$, respectively.

The case of $\mathfrak{s p}(2 k) \oplus \mathfrak{s p}(2 n)$ is analogous.

Thus, the statement is proved.

8.2. Proof of Proposition 4.4.1. Proposition 3.2.2 and Lemmas 4.1 and 4.2 imply that a solution of CYBE corresponding to the order $W \subset \mathbb{D}_{\alpha_{n}}$ induces a subalgebra $L \subset \mathfrak{o}(2 n)$, such that $L+P_{\alpha}^{-}=\mathfrak{o}(2 n)$ and an $L$-invariant Lagrangian subspace in $V_{\alpha, 1}=\mathbb{C}^{2 n}$.

It is well-known that the set of Lagrangian subspaces in $\mathbb{C}^{2 n}$ is a manifold with two connected components and $S O(2 n)$ acts transitively on each component. One of them is generated by $P_{\alpha, 1}^{+}$, and the other one is generated by the following Lagrangian subspace in $\mathbb{C}^{2 n}$ having a 1-dimensional intersection with $P_{\alpha, 1}^{+}$:

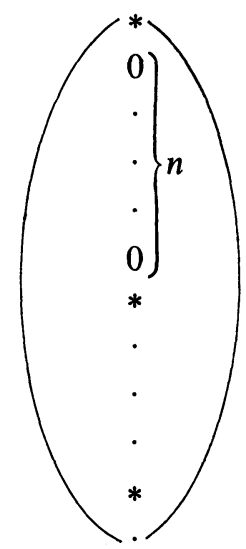

Let $V_{0}$ belongs to the component generated by $P_{\alpha, 1}^{+}$. Then there exists $X \in S O(2 n)$ such that $X^{-1} V_{0} X=P_{\alpha, 1}^{+}$. Hence, $X^{-1} L X \subset P_{\alpha}^{+}, L+P_{\alpha}^{-}=\mathfrak{o}(2 n)$ and $P_{\alpha}^{+}+P_{\alpha}^{-}=\mathfrak{o}(2 n)$ Lemma 4.2 from [15] shows that we can choose $X$ from $G\left(P_{\alpha}^{-}\right)$.

The statement of proposition follows from the Remark to Proposition 3.2.2.

Similarly, in the second case $W \subset \mathbb{D}_{\alpha_{1}}$.

8.3. Proof of Proposition 4.5. As in the proof of Proposition 4.4.1 we can assume that the $r$-dimensional $L$-invariant subspace in $\mathbb{C}^{2 n+1}$ is generated by $e_{1}, e_{2 n}, e_{2 n-1}, \ldots, e_{2 n+2-r}$, where $\left\{e_{i}\right\}$ is the standard basis of $\mathbb{C}^{2 n+1}$. Then the isotropic order $W \subset \mathbb{D}_{1}$ corresponding to $L$ is contained in $\mathbb{D}_{0}$ if $r=1$ and in $\boldsymbol{D}_{r}$ if $r>1$.

8.3.1. Corollary. If $L$ is solvable the corresponding solution is gauge equivalent to a constant one.

8.4. Proof of Corollary 4.6. Proposition 4.4.1 shows that we have to consider only the solutions corresponding to $\alpha_{1}$, where $\alpha_{1} \in D(\mathfrak{o}(5))$ is the singular vertex. Dimensional considerations show that the dimension of $L \subset \mathfrak{o}(5)$ must be odd. Let us consider two cases.

1) First suppose there are no nontrivial isotropic $L$-invariant subspaces. The case when $L$ is irreducible is analyzed by means of Table 1 from Appendix 1 . If $L$ is 
reducible and $V \subset \mathbb{C}^{5}$ is $L$-invariant then the inner product on $V$ is nondegenerate and $\mathbb{C}^{5}=V \oplus V^{\perp}$ (cf. proof of Proposition 4.3). Moreover $\operatorname{dim} V \neq 2$ (otherwise $V$ would contain two isotropic $L$-invariant 1 -dimensional subspaces). Thus, we may assume that $\operatorname{dim} V=4$ and $L$ is an irreducible subalgebra of $\mathfrak{o}(4)$. Then $L=\mathfrak{o}(4)$ and it is impossible because $\operatorname{dim} L$ must be odd.

2) Now suppose $\mathbb{C}^{5}$ contains nontrivial isotropic $L$-invariant subspaces. If the solution is nonconstant then these subspaces cannot be 1-dimensional by Proposition 4.4.1. Then we proceed just as in [15].

9.1.1. Proof of Proposition 5.2.1. Notice that $L_{\alpha}+\varepsilon V_{\alpha, 1}+\varepsilon^{2} V_{\alpha, 2}+\varepsilon^{3} L_{\alpha}=T+T^{*}$, where $T=L_{\alpha}+\varepsilon V_{\alpha, 1}$. Now let $S$ be the projection of $X_{W}$ to $L_{\alpha}+\varepsilon V_{\alpha, 1}$. Clearly, $S$ is closed with respect to the bracket in the affine algebra $\left(L_{\alpha}, V_{\alpha, 1}\right)$ and $S+\left(P_{\alpha}^{-}, P_{\alpha, 1}^{-}\right)=\left(L_{\alpha}, V_{\alpha, 1}\right)$. Let $S^{\perp} \subset T^{*}$ be the annihilator of $S$. Then $X_{W} \supset S^{\perp}$, since $X_{W}$ is a Lagrangian subspace in $L_{\alpha}+\underset{\tilde{X}}{\varepsilon} V_{\alpha, 1}+\varepsilon^{2} V_{\alpha, 2}+\varepsilon^{3} L_{\alpha}$ and $X_{W} \subset S+T^{*}$. Hence, $X_{W}$ is uniquely recovered from $\tilde{X}_{W}^{\alpha, 1} \subset S+T^{*} / S^{\perp}=S+S^{*}$. Clearly, the image of $\widetilde{X}_{W}$ under the projection $S+S^{*} \rightarrow S$ is $S$ and $\tilde{X}_{W}$ is uniquely recovered from a skewsymmetric form $B$, as follows:

$$
\tilde{X}_{W}=\left\{x+f(x): x \in S ; f(x) \in S^{*} ; f(x)(y)=B(x, y)\right\} .
$$

Then $X_{W}=\left\{x+f(x)+t: t \in S^{\perp}\right\}$. It remains to find conditions under which $X_{W}$ is a subalgebra. Clearly, $X_{W} \cap\left(\varepsilon^{2} V_{\alpha, 2}+\varepsilon^{3} L_{\alpha}\right)=S^{\perp}$, and $S^{\perp}$ is an ideal in $X_{W}$. Since $\varepsilon^{2} V_{\alpha, 2}+\varepsilon^{3} L_{\alpha}$ is a commutative Lie algebra, $S^{\perp}$ is $S$-module. Let $x, y \in S$. Define $[x, y]_{V_{\alpha, 2}}$ setting $[x, y]=[x, y]_{\mathrm{af}}+[x, y]_{V_{\alpha, 2}}$. Then $[x+f(x), y+f(y)]=[x, y]_{\mathrm{af}}+$ $[x, f(y)]+[f(x), y]+[x, y]_{V_{\alpha, 2}} \bmod S^{\perp}$. For any $z \in S$ we have $\left.\left(f[x, y]_{\mathrm{af}}\right), z\right)=$ $([x, f(y)], z)+([f(x), y], z)+\left([x, y]_{V_{\alpha, 2}}, z\right)$. Clearly, this is equivalent to the desired statement. Since our arguments are easily invertible, we have proved that any Lagrangian subalgebra in $L_{\alpha}+\varepsilon V_{\alpha, 1}+\varepsilon^{2} V_{\alpha, 2}+\varepsilon^{3} L_{\alpha}$ is of the form $(S, B)$.

Let us prove that the condition

$$
X_{W} \cap\left(P_{\alpha}^{-}+\varepsilon^{3}\left(P_{\alpha}^{-}\right)^{\perp}+\varepsilon P_{\alpha, 1}^{-}+\varepsilon^{2} P_{\alpha, 2}^{-}\right)=0
$$

is equivalent to the nondegeneracy of $B$ on $S \cap\left(P_{\alpha}^{-}, P_{\alpha, 1}^{-}\right)$. Indeed, $P_{\alpha}^{-}+\varepsilon^{3}\left(P_{\alpha}^{-}\right)^{\perp}+$ $\varepsilon P_{\alpha, 1}^{-}+\varepsilon^{2} P_{\alpha, 2}^{-}=\left(P_{\alpha}^{-}, P_{\alpha, 1}^{-}\right)+\left(P_{\alpha}^{-}, P_{\alpha, 1}^{-}\right)^{\perp}$. Let $s+f(s)+s^{\perp}=p+p^{\perp}$, where $s \in S$; $f(s) \in S^{*} ; s^{\perp} \in S^{\perp} ; p \in\left(P_{\alpha}^{-}, P_{\alpha, 1}^{-}\right) ; p^{\perp} \in\left(P_{\alpha}^{-}, P_{\alpha, 1}^{-}\right)^{\perp}$. Then

$$
s=p \in S \cap\left(P_{\alpha}^{-}, P_{\alpha, 1}^{-}\right)
$$

and

$$
f(s) \in S^{\perp}+\left(P_{\alpha}^{-}, P_{\alpha, 1}^{-}\right)^{\perp}=\left(S \cap\left(P_{\alpha}^{-}, P_{\alpha, 1}^{-}\right)\right)^{\perp},
$$

which means that $f(s)(x)=B(s, x)=0$ for any $x \in S$. Our statement is completely proved.

10. Proof of Proposition A.2.

1) Analysis of Table 1.

2) It follows from Propositions 4.4 and 4.5.

3) We must check that $L \cap P_{1}$ is a Frobenius algebra (by Theorem 2.1). It follows by methods of [15]. 
Acknowledgements. I am thankful to V. Drinfeld, who posed the problem, and D. Leites for help.

\section{References}

1. Arnold, V. I., Ilyashenko, Yu. S.: Ordinary differential equations. Modern Problems of Math., Fund. Trends. v.1 (Results of Sci. and Technology). Moscow VINITI, 1985 (in Russian)

2. Belavin, A., Drinfeld, V.: On classical Yang-Baxter equation for simple Lie algebras. Funct. Anal. Appl. 16, 1-29 (1982) (in Russian)

3. Belavin, A., Drinfeld, V.: On classical Yang-Baxter equation for simple Lie algebras. Funct. Anal. Appl. 17 (3), 69-70 (1983) (in Russian)

4. Bourbaki, N.: Groups et algebras de Lie. Paris: Hermann 1975

5. Elashvili, A.: Stationary subalgebras of generic points for irreducible linear Lie algebras. Sov. J. Funct. Anal. 6 (1), 51-62, 6 (2), 65-78 (1972)

6. Elashvili, A.: Frobenius Lie algebras. Funct. Anal. and Appl. 16 (4), 94-95 (1982); II. Proc. Math. Inst. Georgia Acad. Sci., 1986, pp.126-137 (in Russian)

7. Onishchik, A., Vinberg, E.: Seminar on algebraic groups and Lie groups. Berlin, Heidelberg, New York: Springer 1990

8. Serre, J.-P.: Letter to V. Drinfeld, September 12, 1985

9. Soule, C.: Chevalley groups over polynomial rings. In: Homological Group Theory, London MS, Lect. Notes vol. 36. Cambridge 1979

10. Spiz, G.: Classification of irreducible locally transitive Lie groups. In: Geometric methods in problems of analysis and algebra. Yaroslavl. University Press, Yaroslavl, 1978, 152-160 (in Russian and in Appendix to [15] in English)

11. Stolin, A.: Classical Yang-Baxter equation and Frobenius Lie algebras. In: Proceedings of the XIX All-Union algebraic conference. Lvov, 9-11 September, 267. Lvov: Lvov University Press 1987 (in Russian)

12. Stolin, A.: On classical Yang-Baxter equation. In: Topological algebra, 69. Kishinev: Shtinitsa 1988 (in Russian)

13. Stolin, A.: Rational solutions of classical Yang-Baxter equation and quasi-Frobenius subalgebras in sl(3). In: Proceedings of the International algebraic conference. These of talks on the theory of rings, algebras and modules. Novosibirsk: Novosibirsk University Press, 131,1989 (in Russian)

14. Stolin, A.: On a degree of a rational solution of the classical Yang-Baxter equation. In: Proceedings of the international algebraic conference. Thesis of talks on the theory of rings, algebras and modules, 132. Novosibirsk: Novosibirsk University Press 1989 (in Russian)

15. Stolin, A.: On rational solutions of Yang-Baxter equation for $s \mathfrak{l}(n)$. Math. Scand. (to appear)

16. Vinberg, E., Kimmelfeld, B.: Homogeneous domains on flag manifolds and spherical subgroups of semisimple Lie groups. Sov. J. Funct. Anal. 12 (3), 12-19 (1978) 